\title{
Endovascular Treatment of Acute Ischemic Stroke in Patients Younger than 18 Years
}

\author{
Sibasankar Dalai ${ }^{1}$ Uday Limaye ${ }^{2}$ Satyarao Kolli ${ }^{3}$ Mohan V. Sumedha Maturu ${ }^{3}$ \\ Randhi Venkata Narayana ${ }^{4}$ Rajesh Pati $^{3}$ Suresh Kumar Korada ${ }^{4}$ Kuchelababu Vankineni ${ }^{5}$ \\ Anantamakula Sameera ${ }^{6}$ Aravind Varma Datla ${ }^{7}$
}

${ }^{1}$ Department of Interventional Neuroradiology, Medicover Hospitals,

Visakhapatnam, Andhra Pradesh, India

2 Department of Neurointervention, Lilavati Hospital, Mumbai,

Address for correspondence Sibasankar Dalai, MD, FACP, FINR, 1st Floor OPD - 14, Medicover Hospitals, Plot No.5 - Health City Arilova,

Maharashtra, India

3 Department of Neurology, Medicover Hospitals, Visakhapatnam,

Andhra Pradesh, India

${ }^{4}$ Department of Neurology, Seven Hills Hospitals, Visakhapatnam,

Andhra Pradesh, India

${ }^{5}$ Department of Anaesthesiology and Critical Care, Seven Hills

Hospitals, Visakhapatnam, Andhra Pradesh, India

${ }^{6}$ Department of Radiodiagnosis, Medicover Hospitals,

Visakhapatnam, Andhra Pradesh, India

${ }^{7}$ Department of Internal Medicine, Medicover Hospitals,

Visakhapatnam, Andhra Pradesh, India

J Clin Interv Radiol ISVIR

\begin{abstract}
Keywords

- stroke in young

- mechanical thrombectomy

- thromboaspiration

Rapid and effective revascularization is very important in the treatment of acute ischemic stroke (AIS). Endovascular treatment is a promising modality in the management of AIS in young patients. We evaluated the clinical and imaging records in 14 patients younger than 18 years presenting within 6 hours of AIS. They received endovascular therapy (ET) either by mechanical thrombectomy, thromboaspiration, or both (Solumbra) between July 2017 and June 2021 in our institute. The National Institute of Health Stroke Scale (NIHSS) score was calculated on admission and before the discharge of all patients. The 90-day modified Rankin Scale (mRS) score on disability-free outcome was also evaluated. The mean preprocedure NIHSS score was $10.78 \pm 2.11$ that improved to $4.5 \pm 1.88$ after the procedure. Thrombolysis in cerebral infarction ( $\mathrm{TICl}$ ) grade $2 \mathrm{~b}$ and 3 blood flow could be established in 12 (85.72\%) patients. One patient had $\mathrm{TICl} 2 \mathrm{a}$ flow and one patient had recurrent occlusion despite repeated recanalization ( $\mathrm{TICl}$ grade 0$)$. The disability-free outcome, mRS score at 90 days was 0 to 1 in 12 (85.72\%) patients, mRS score 2 in one (7.14\%) patient, and mRS score 3 in one patient (7.14\%). We did not have any major complication related to the procedure. ET provides high rates of arterial recanalization and favorable disability-free outcomes in young patients with AIS.
\end{abstract}

DOI https://doi.org/ 10.1055/s-0041-1740568. ISSN 2457-0214.
(C) 2021. Indian Society of Vascular and Interventional Radiology. All rights reserved.

This is an open access article published by Thieme under the terms of the Creative Commons Attribution-NonDerivative-NonCommercial-License, permitting copying and reproduction so long as the original work is given appropriate credit. Contents may not be used for commercial purposes, or adapted, remixed, transformed or built upon. (https://creativecommons.org/ licenses/by-nc-nd/4.0/)

Thieme Medical and Scientific Publishers Pvt. Ltd., A-12, 2nd Floor, Sector 2, Noida-201301 UP, India 


\section{Introduction}

Strokes are one of the preeminent causes of disability and death worldwide and are a significant health burden. In India, the cumulative incidence of stroke ranges from 105 to 152 per 100,000 population per year. The crude prevalence of stroke ranges from 44.29 to 559 per 100,000 population in different parts of our country. ${ }^{1}$ It is estimated that $41 \%$ of all deaths due to noncommunicable diseases are due to stroke. Stroke is responsible for $72 \%$ of the disability-adjusted life years. $^{2}$

Stroke can occur in teenagers, children, and newborns. The risk of stroke in children is most significant in the first year of life. Strokes remain among the top 10 causes of death in children. A stroke happens in approximately 1 in 4,000 live births. The risk of stroke from birth through age 18 years is almost 11 in 100,000 children per year. Diagnosis of stroke in this population is highly challenging because approximately $50 \%$ of children afflicted have no known vascular risk factor. ${ }^{3}$ Common risk factors for stroke in children include sickle cell disease, clotting disorders, congenital heart defects, immune disorders, arterial diseases, focal cerebral arteriopathy (one of the most common causes of large vessel occlusion [LVO] in previously healthy children), head or neck trauma, preeclampsia, and eclampsia in the mother.

Intracranial artery occlusion accounts for approximately $80 \%$ of all acute ischemic strokes (AISs) and usually manifests as focal neurologic deficits. Prompt revascularization using recombinant tissue plasminogen activator ( $t-P A)$ is the mainstay for the treatment of AIS. ${ }^{4}$ However, revascularization rates are often suboptimal, especially in the internal carotid artery and middle cerebral artery (MCA) occlusions (LVO). ${ }^{5}$ Furthermore, delayed patient presentation due to poor awareness of stroke makes the patient ineligible for intravenous thrombolysis. ${ }^{4}$

Alternative recanalizing approaches such as endovascular therapy (ET) are helpful in these situations. ET has been tried for many years and has been progressively gaining favor in the management of AIS. There has been a notable transition from intra-arterial thrombolysis using drugs such as prourokinase to more advanced procedures such as mechanical thrombectomy (MT) using devices to remove the thrombus. ${ }^{6}$

In 2015 and 2016, six randomized controlled trials focused on the endovascular treatment of AIS patients caused by LVO were published in Lancet Neurology and The New England Journal of Medicine. All these trials favor thrombectomy for AIS patients with LVO, which can significantly improve patients' 90-day outcomes.

1. THRACE: Mechanical thrombectomy after intravenous alteplase versus alteplase alone after stroke.

2. MR CLEAN: A multicenter randomized clinical trial of endovascular treatment for acute ischemic stroke in the Netherlands.

3. ESCAPE: Randomized assessment of rapid endovascular treatment of ischemic stroke.

4. SWIFT PRIME: Solitaire with the intention for thrombectomy as primary endovascular treatment.
5. REVASCAT: Endovascular revascularization with Solitaire device versus best medical therapy in anterior circulation stroke within 8 hours.

6. EXTEND-IA: Endovascular therapy for ischemic stroke with perfusion-imaging studies.

The data on the efficacy and safety of endovascular treatment for AIS in adults are promising. However, the data on these AIS interventions in patients younger than 18 years is limited. ${ }^{6-14}$

\section{Materials and Methods}

We evaluated the medical records of patients younger than 18 years, with a diagnosis of AIS presenting within 6 hours of onset, who underwent ET for AIS between July 2017 and June 2021. All clinical parameters including presenting signs and symptoms, imaging, National Institute of Health Stroke Scale (NIHSS) scores were studied. Clinical outcomes, discharge summaries, and follow-up modified Rankin Scale (mRS) score at 90 days were recorded.

We excluded stroke mimics like Todd's paralysis after a seizure, complex migraine, pseudoseizures, and conversion disorders. Computed tomography (CT) brain with CT angiogram or magnetic resonance imaging of the brain, including diffusion, apparent diffusion coefficient, fluid-attenuated inversion recovery, and susceptibility, and magnetic resonance angiogram of the neck and cerebral vessels were done.

Relevant blood investigations, including complete blood count, renal function test, liver function test, and coagulation profile, were done and shifted the patients to an angiographic suite for a cerebral angiogram and possible endovascular treatment. Patients were given detailed information about the endovascular treatment options, including their possible outcomes and complications before initiating the treatment. Consent for the procedure was obtained from the parent or guardian. ET (MT/thromboaspiration $[\mathrm{TA}]$ ) was done.

\section{Mechanical Thrombectomy}

A cerebral angiogram was done from the right transfemoral arterial approach after securing a 6F short sheath. An angiogram of both the internal carotid artery and the vertebral artery was done. The occluded vessel was cannulated with a 6 F guiding catheter (Cordis Inc.), and an angiogram was done (-Fig. 1). The thrombus was crossed with a microcatheter, Rebar 0.27 (Medtronics Inc.), the wire was removed, and a $4 \times 20 \mathrm{~mm}$ Solitaire (Medtronics Inc.) or a $4 \times 30 \mathrm{~mm}$ Trevo (Stryker Neurovascular Inc.) was fully deployed across the thrombus. The stent was left open for 5 minutes. The distal flow was established. The stent was removed with simultaneous aspiration from the side port of the Y-connector at the guiding catheter hub. A check angiogram was performed to check the flow in the occluded vessel (-Fig. 2). If suboptimal recanalization was seen, the procedure was repeated. In some patients, a combination of aspiration and stent retriever (Solumbra) was used. 


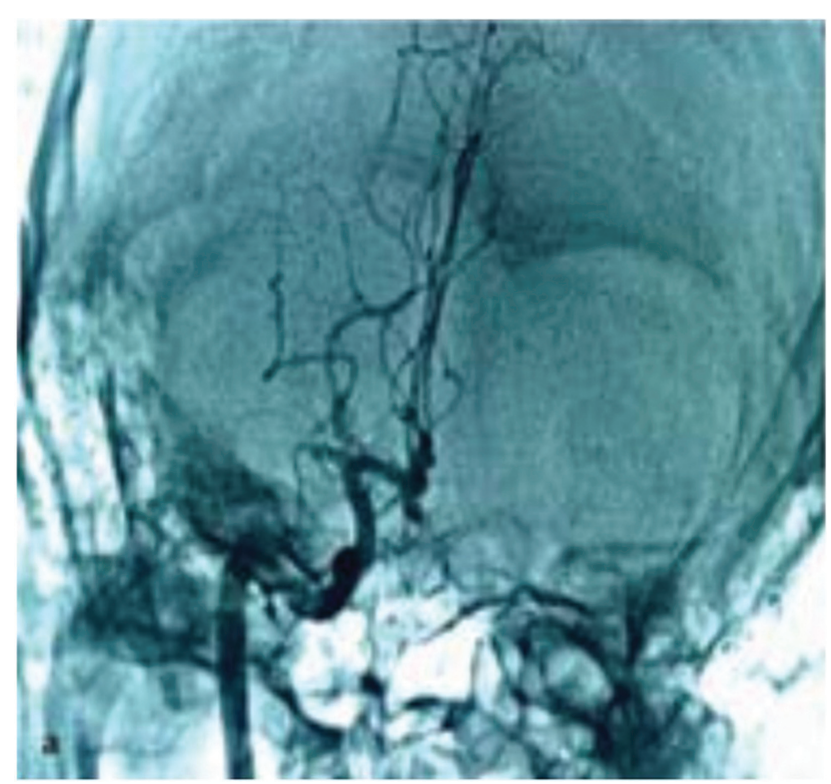

Fig. 1 Cerebral angiogram of the patient showing occlusion of right middle cerebral artery (MCA) M1.

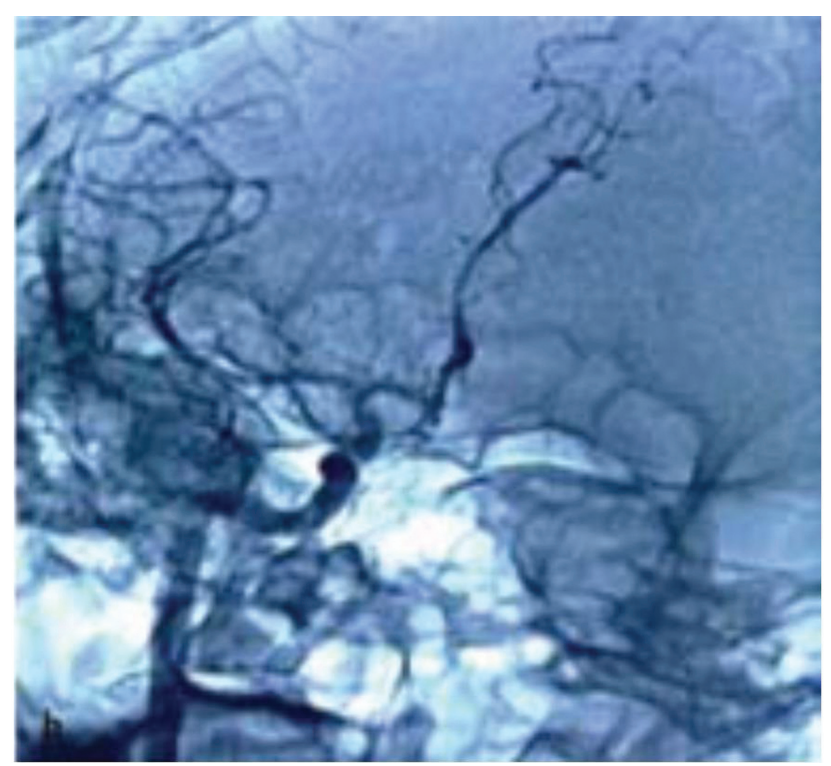

Fig. 2 Postthrombectomy angiogram demonstrating complete recanalization of the middle cerebral artery (MCA)-M1.

\section{Thromboaspiration}

Standard procedure for cerebral angiogram was followed. The occluded vessel was cannulated. A 6F long sheath (Cook Medical) was placed in the proximal course of the vessel. An angiogram was performed (-Fig. 3). A 6F CT aspiration catheter (Stryker Neurovascular Inc.) with 5.4F distal lumen was navigated with an SL10 microcatheter and Synchro 0.014 wire (Stryker Neurovascular Inc.) inside the lumen of CAT6. The thrombus was crossed with SL10 and Synchro. The aspiration catheter was placed at the proximal end of the thrombus. The SL10 and Synchro were removed, and aspiration was performed with an aspiration pump. Simultaneous aspiration was performed from the side port of the 7F long

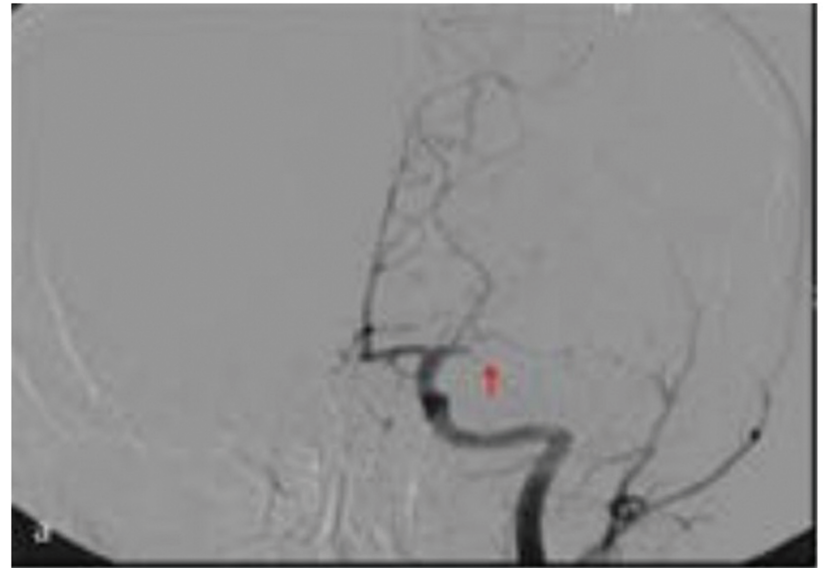

Fig. 3 Anteroposterior (AP) view of cerebral angiogram in an acute ischemic stroke (AIS) patient, demonstrating occlusion of left middle cerebral artery (MCA)-M1. Red arrow: Site of occlusion.

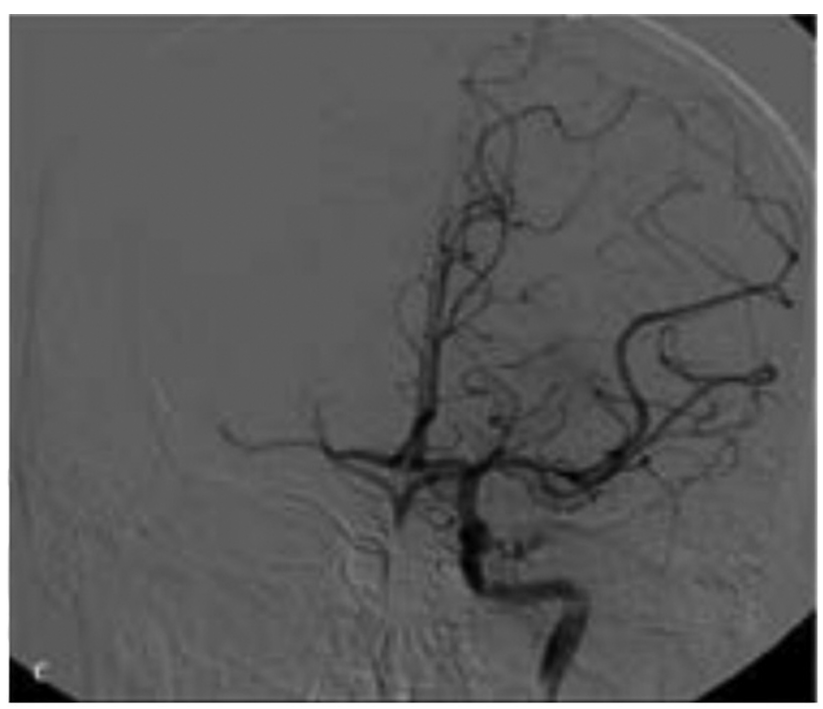

Fig. 4 Anteroposterior (AP) view of the cerebral angiogram of the same patient after thromboaspiration showing complete recanalization of the middle cerebral artery (MCA).

sheath. Check angiogram was performed to check the flow in the occluded vessel (-Fig. 4). If suboptimal recanalization was seen, the procedure was repeated. In some patients, Solumbra was used.

The patient was shifted to the acute stroke care unit for further management. Investigations including thrombophilia profile, sickling test, homocysteine levels, and proteins $\mathrm{C}$ and $\mathrm{S}$ deficiency were done to evaluate the cause for stroke in young.

All data were entered electronically and presented as mean and standard deviation for continuous variables. We performed statistical analysis using SPSS software 20.0 (Statistical Package for the Social Sciences; IBM Corp.).

\section{Results}

Fourteen patients out of whom nine were male, ranging from 6 to 17 years (mean age 11.64 years), were included in this 
Endovascular Treatment of Acute Ischemic Stroke in Patients Younger than 18 Years Dalai et al.

Table 1 Demographic characteristics and treatment details of patients

\begin{tabular}{|c|c|c|c|c|c|c|c|c|}
\hline Patient no. & Age (y) & Sex & $\begin{array}{l}\text { Vessel } \\
\text { occluded }\end{array}$ & $\begin{array}{l}\text { Recanalizing } \\
\text { technique used }\end{array}$ & $\begin{array}{l}\text { Window } \\
\text { period (in h) }\end{array}$ & $\begin{array}{l}\text { NIHSS at } \\
\text { presentation } \\
\text { (before ET) }\end{array}$ & $\begin{array}{l}\text { NIHSS at } \\
\text { the time } \\
\text { of discharge } \\
\text { (after ET) }\end{array}$ & $\mathrm{mRS}$ at $90 \mathrm{~d}$ \\
\hline 1 & 6 & $\mathrm{~F}$ & MCA & MT & 3 & 14 & 5 & 0 \\
\hline 2 & 12 & $\mathrm{M}$ & ICA & MT & 5 & 12 & 9 & 3 \\
\hline 3 & 14 & $\mathrm{~F}$ & MCA & Solumbra & 6 & 9 & 2 & 0 \\
\hline 4 & 15 & $M$ & MCA & $\mathrm{TA}$ & 4.5 & 15 & 6 & 2 \\
\hline 5 & 17 & $M$ & ICA & TA & 3.5 & 10 & 2 & 0 \\
\hline 6 & 16 & $M$ & ICA & MT & 5 & 8 & 4 & 0 \\
\hline 7 & 11 & $\mathrm{~F}$ & MCA & MT & 4 & 11 & 5 & 0 \\
\hline 8 & 11 & M & MCA & MT & 3 & 9 & 4 & 0 \\
\hline 9 & 8 & $\mathrm{M}$ & MCA & TA & 2.5 & 10 & 4 & 0 \\
\hline 10 & 9 & $M$ & MCA & Solumbra & 3 & 8 & 3 & 0 \\
\hline 11 & 7 & $M$ & MCA & MT & 4 & 9 & 6 & 1 \\
\hline 12 & 13 & $\mathrm{~F}$ & ICA & TA & 3 & 12 & 5 & 1 \\
\hline 13 & 15 & $M$ & ICA & MT & 5 & 11 & 2 & 0 \\
\hline 14 & 9 & $\mathrm{~F}$ & MCA & Solumbra & 4.5 & 13 & 6 & 1 \\
\hline
\end{tabular}

Abbreviations: ET, endovascular therapy; F, female; ICA, internal carotid artery; M, male; MCA, middle cerebral artery; mRS, modified Rankin Scale; MT, mechanical thrombectomy; NIHSS, National Institute of Health Stroke Scale; TA, thromboaspiration.

study. The demographic characters and the treatment details are summarized in - Table $\mathbf{1}$.

The mean NIHSS scores improved from $10.78 \pm 2.11$ before the procedure to $4.5 \pm 1.88$ after the procedure. Revascularization (thrombolysis in cerebral infarction [TICI] flow $2 \mathrm{~b}$ and 3 ) following the ET procedure was established in 12 patients. A TICI grade 2a blood flow was established in one patient. One patient had recurrent occlusion despite recanalization (TICI grade 0 ).

\section{Discussion}

Treatment for pediatric stroke should focus on early recanalization followed by treating the specific cause. For children younger than 18 years, t-PA is controversial and currently limited to clinical trials. Further studies on the safety and benefits of this treatment are necessary. However, the endovascular stroke treatment is getting safer and effective and must be explored for its role in treatment in pediatric stroke.

Currently, pediatric stroke management is limited to controlling blood pressure, maintaining normal body temperature, proper hydration, normal blood sugar levels, treating seizures, and transfusing blood for sickle cell patients. However, in pediatric stroke patients with LVO, we offered endovascular treatment for early recanalization.

The clinical outcomes following pediatric AIS are poor, with devastating effects on the child and their prospects. A sizable portion of the patients develops long-lasting neurological issues such as epilepsy, behavioral problems, motor dysfunction, speech and language impairments, deficits in attention, complex cognitive skills, executive function, working memory, and processing speed.
Endovascular treatment of AIS in large artery occlusion in the proximal anterior circulation with second-generation MT devices is safe and effective for reducing disability. ${ }^{15-18}$ Five multicenter, open-label randomized controlled trials (MR CLEAN, ${ }^{19}$ ESCAPE, $^{5}$ SWIFT PRIME, ${ }^{20}$ EXTEND-IA, ${ }^{21}$ and REVASCAT $^{22}$ ) demonstrate the efficacy of endovascular treatment. MT was beneficial across various patient subgroups, including patients aged 80 years or older, high initial stroke severity, and those not treated with intravenous alteplase. Though many studies have explored the stroke profile and management outcomes in adult patients, pediatric stroke remains an underrecognized entity despite being an important cause of lifelong disability. Recanalization is an essential indicator of therapeutic benefit in the early-phase thrombolytic treatment with a strong positive correlation with improved functional outcomes and reduced mortality in the management of AIS in adults and children. ${ }^{12}$

In younger children, a concern may be that the usage of ET devices may not be appropriate for the pediatric intracranial arterial architecture because of size issues. However, it has been shown that the cranial growth is rapid during the early years of life and then slows markedly with virtually complete growth by the age of 6 years, and $85 \%$ of growth is complete by the age of $5 .^{23}$ Similarly, by the age of 4 years, the intracranial arteries have reached 81 to $99 \%$ of their adult diameters with $87 \%$ of the MCA diameter reached by 6 months of age. ${ }^{24}$ Therefore, from a practical point of view, these procedures in children aged 4 to 5 years and older require only a slight change, such as using smaller guide catheters, and should not pose a significant challenge for experienced interventional neuroradiologists. ${ }^{25,26}$ 
Though there are no large-scale case-controlled trials or safety studies, there is a heap of information in the form of well-reported case reports and series suggesting that selective pediatric patients may benefit significantly from ET. Barry et al reported that a well-versed multidisciplinary team could contribute to improved outcomes using ET. ${ }^{27} \mathrm{~A}$ systematic retrospective review by Bhatia et al revealed that 90.6\% had good long-term neurological outcomes (mRS score $0-2$ ). Out of 110 pediatric patients, symptomatic intracranial hemorrhage and death occurred in 1 and 2 patients, respectively. ${ }^{28}$ In a series of five cases published by Bhatia et al, mRS scores of 0 to 1 were achievable in all the patients with no periprocedural complications. ${ }^{28}$ Similarly, a retrospective study of 12 children by Sporns et al across three German tertiary care stroke centers declared good neurological outcomes with no complications in the treated patients. ${ }^{29}$ These findings are comparable to ours and once again emphasize the usefulness of ET, particularly thrombectomy.

In an institutional review by Cappellari et al, the favorable outcomes of primary MT were superior to secondary MT (69\% vs. $43 \%)^{30}$

In contrast, Fragata et al noticed improvements in only $57 \%$ of the subjects. ${ }^{31}$ In the study by Sun et al, of 11 children aged below 5 years, favorable outcomes were achieved in $64 \%$ of the patients. ${ }^{32}$ The reasons for these observations could be the time lag in the initiation of treatment and the relatively younger age of the participants. ${ }^{31,32}$ These findings once again reinforce our belief that early treatment and increasing age of the child (whose intracranial vasculature more closely resembles the adult, thereby providing a sense of familiarity and using standard equipment) are indicators for favorable neurological outcomes.

MT can be associated with access site hematoma, pseudoaneurysm, arterial perforation, and arterial dissection. $5,20-22$ Vasospasm is uncommonly seen during thrombectomy or TA. It is treated with intraprocedural, intra-arterial nimodipine infusion. However, endovascular treatment is not associated with increased rates of symptomatic intracranial hemorrhage or mortality. In a metaanalysis of five trials, with pooled patient-level data for 1,287 patients, there was no significant difference between the intervention population and control population for 90-day symptomatic intracranial hemorrhage $(4.4 \%$ vs. $4.3 \%)$ or mortality (15\% vs. $19 \%)^{33}$

A case series reported that the application of conventional techniques and equipment developed for adults can be used in children as young as 5 years and that their mRS scores at 90 days after the procedure improved significantly. ${ }^{9,10}$

In our study of six patients, the recanalization rates are reasonable. NIHSS and mRS have improved significantly postrecanalization. One patient had recurrent occlusion, and we treated him successfully.

After early recovery from stroke, all patients were evaluated to determine the risk factor for stroke in young. They underwent

1. Sickling test.

2. Coagulation profile.

\section{Two-dimensional echocardiography.}

A detailed family history of stroke and cerebrovascular disease was taken. We found proteins C and S deficiency in two patients. One patient had sickle cell disease. After the ET, we treated the patients and followed according to their risk profile.

ET in children with AIS is safe and efficacious and can result in high rates of successful recanalization. In addition, there was a remarkable improvement in these patients' outcomes, implying that endovascular procedures positively impact the functional outcome of these patients. However, these findings need to be tested in a more significant number of patients.

\section{Conclusion}

Endovascular techniques including MT, intra-arterial thrombolysis, and TA are safe and efficacious for achieving recanalization in patients younger than 18 years with AIS. These procedures also can extend the window period for treatment. However, further large-scale interventional studies are required for the confirmation of the findings in this study.

\section{Conflict of Interest}

None declared.

\section{References}

1 Kamalakannan S, Gudlavalleti ASV, Gudlavalleti VSM, Goenka S, Kuper $\mathrm{H}$. Incidence \& prevalence of stroke in India: a systematic review. Indian J Med Res 2017;146(02):175-185

2 Biswas T. India unveils plans to improve stroke research and care. Lancet 2013;381(9862):190

3 Mallick AA, Ganesan V, Kirkham FJ, et al. Childhood arterial ischaemic stroke incidence, presenting features, and risk factors: a prospective population-based study. Lancet Neurol 2014;13 (01):35-43

4 Khalessi AA, Fargen KM, Lavine S, Mocco J. Commentary: societal statement on recent acute stroke intervention trials: results and implications. Neurosurgery 2013;73(02):E375-E379

5 Goyal M, Demchuk AM, Menon BK, et al; ESCAPE Trial Investigators. Randomized assessment of rapid endovascular treatment of ischemic stroke. N Engl J Med 2015;372(11):1019-1030

6 Mijajlovic MD. Thrombolytic or endovascular therapy for acute ischemic stroke: time is brain. J Neurosci Rural Pract 2014;5(01): 3-5

7 Nogueira RG, Lutsep HL, Gupta R, et al; TREVO 2 Trialists. Trevo versus Merci retrievers for thrombectomy revascularisation of large vessel occlusions in acute ischaemic stroke (TREVO 2): a randomised trial. Lancet 2012;380(9849):1231-1240

8 Goeggel Simonetti B, Cavelti A, Arnold M, et al. Long-term outcome after arterial ischemic stroke in children and young adults. Neurology 2015;84(19):1941-1947

9 Zanaty M, Chalouhi N, Starke RM, et al. Endovascular stroke intervention in the very young. Clin Neurol Neurosurg 2014; 127:15-18

10 Bodey C, Goddard T, Patankar T, et al. Experience of mechanical thrombectomy for paediatric arterial ischaemic stroke. Eur J Paediatr Neurol 2014;18(06):730-735

11 Chalouhi N, Tjoumakaris S, Starke RM, et al. Endovascular stroke intervention in young patients with large vessel occlusions. Neurosurg Focus 2014;36(01):E6 
12 Wolpert SM, Bruckmann H, Greenlee R, Wechsler L, Pessin MS, del Zoppo GJ. Neuroradiologic evaluation of patients with acute stroke treated with recombinant tissue plasminogen activator. The rt-PA Acute Stroke Study Group. AJNR Am J Neuroradiol 1993;14(01):3-13

13 Saposnik G, Guzik AK, Reeves M, Ovbiagele B, Johnston SC. Stroke prognostication using age and NIH Stroke Scale: SPAN-100. Neurology 2013;80(01):21-28

14 Natarajan SK, Snyder KV, Siddiqui AH, Ionita CC, Hopkins LN, Levy EI. Safety and effectiveness of endovascular therapy after 8 hours of acute ischemic stroke onset and wake-up strokes. Stroke 2009; 40(10):3269-3274

15 Campbell BCV, Donnan GA, Lees KR, et al. Endovascular stent thrombectomy: the new standard of care for large vessel ischaemic stroke. Lancet Neurol 2015;14(08):846-854

16 Touma L, Filion KB, Sterling LH, Atallah R, Windle SB, Eisenberg MJ. Stent retrievers for the treatment of acute ischemic stroke: a systematic review and meta-analysis of randomized clinical trials. JAMA Neurol 2016;73(03):275-281

17 Tsivgoulis G, Katsanos AH, Mavridis D, Magoufis G, Arthur A, Alexandrov AV. Mechanical thrombectomy improves functional outcomes independent of pretreatment with intravenous thrombolysis. Stroke 2016;47(06):1661-1664

18 Saver JL, Goyal M, van der Lugt A, et al; HERMES Collaborators. HERMES Collaborators. Time to treatment with endovascular thrombectomy and outcomes from ischemic stroke: a metaanalysis. JAMA 2016;316(12):1279-1288

19 Berkhemer OA, Fransen PS, Beumer D, et al; MR CLEAN Investigators. A randomized trial of intraarterial treatment for acute ischemic stroke. N Engl J Med 2015;372(01):11-20

20 Saver JL, Goyal M, Bonafe A, et al; SWIFT PRIME Investigators. Stent-retriever thrombectomy after intravenous t-PA vs. t-PA alone in stroke. N Engl J Med 2015;372(24):2285-2295

21 Campbell BC, Mitchell PJ, Kleinig TJ, et al. EXTEND-IA Investigators. Endovascular therapy for ischemic stroke with perfusionimaging selection. N Engl J Med 2015;372(11):1009-1018

22 Jovin TG, Chamorro A, Cobo E, et al; REVASCAT Trial Investigators. Thrombectomy within 8 hours after symptom onset in ischemic stroke. N Engl J Med 2015;372(24):2296-2306
23 Waitzman AA, Posnick JC, Armstrong DC, Pron GE. Craniofacial skeletal measurements based on computed tomography: part II. Normal values and growth trends. Cleft Palate Craniofac J 1992;29 (02):118-128

24 He L, Ladner TR, Pruthi S, et al. Rule of 5: angiographic diameters of cervicocerebral arteries in children and compatibility with adult neurointerventional devices. J Neurointerv Surg 2016;8 (10):1067-1071

25 Arat YO, Arat A, Aydin K. Angiographic morphometry of internal carotid artery circulation in Turkish children. Turk Neurosurg 2015;25(04):608-616

26 Bhogal P, Hellstern V, AlMatter M, et al. Mechanical thrombectomy in children and adolescents: report of five cases and literature review. Stroke Vasc Neurol 2018;3(04): 245-252

27 Barry M, Hallam DK, Bernard TJ, Amlie-Lefond C. What is the role of mechanical thrombectomy in childhood stroke? Pediatr Neurol 2019;95:19-25

28 Bhatia K, Kortman H, Blair C, et al. Mechanical thrombectomy in pediatric stroke: systematic review, individual patient data metaanalysis, and case series. J Neurosurg Pediatr 2019; $\cdots: 1-14$. Doi: 10.3171/2019.5.PEDS19126

29 Sporns PB, Kemmling A, Hanning U, et al. Thrombectomy in childhood stroke. J Am Heart Assoc 2019;8(05):e011335

30 Cappellari M, Moretto G, Grazioli A, Ricciardi GK, Bovi P, Ciceri EFM. Primary versus secondary mechanical thrombectomy for anterior circulation stroke in children: an update. J Neuroradiol 2018;45(02):102-107

31 Fragata I, Morais T, Silva R, et al. Endovascular treatment of pediatric ischemic stroke: a single center experience and review of the literature. Interv Neuroradiol 2021;27(01):16-24

32 Sun LR, Felling RJ, Pearl MS. Endovascular mechanical thrombectomy for acute stroke in young children. J Neurointerv Surg 2019; 11(06):554-558

33 Goyal M, Menon BK, van Zwam WH, et al; HERMES collaborators. Endovascular thrombectomy after large-vessel ischaemic stroke: a meta-analysis of individual patient data from five randomised trials. Lancet 2016;387(10029):1723-1731 